\title{
The Importance of Aloe debrana Plant as a Thickening Agent for Disperse Printing of Polyester and Cotton in Textile Industry
}

Sisay Awoke ${ }^{1 *}$, Yirga Adugna1, Redwan Jihad², Habtam Getaneh ${ }^{3}$

${ }^{1}$ Department of Chemistry, College of Natural Science, Wollo University, Ethiopia

${ }^{2}$ Department of Textile Engineering, Wollo University, Ethiopia

${ }^{3}$ Department of Textile Biology, Wollo University, Ethiopia

\begin{abstract}
Aloe debrana plant grows in several regions in South Wollo, Ethiopia, and has been used mostly as some of traditional medicine, and to stop breastfeeding. The technical feasibility of using Aloe debrana gel as a new thickener for printing polyester and cotton with disperse dyes was examined in 2013. The results indicated that the properties of the printed fabric samples (colour strength, $\mathrm{K} / \mathrm{S}$, overall fastness properties) were dependent on gel concentration, as the fixation conditions using direct heating technique. The optimum conditions for printing polyester fiber with disperse dyes using Aloe debrana gel as a thickener were as follows: $30 \mathrm{~g} / \mathrm{kg}$ disperse dye, $75 \mathrm{~g} / \mathrm{kg}$ urea, $10 \mathrm{~g} / \mathrm{kg}$ citric acid, $500 \mathrm{~g} / \mathrm{kg}$ Aloe debrana thickener and other water drying at $100^{\circ} \mathrm{C}$ for $3 \mathrm{~min}$ followed by direct fixation for $6 \mathrm{~min}$ at $180^{\circ} \mathrm{C}$. A natural preservative lemon juice has high potential to preserve the gel more than two months. The results showed that no significant difference between fresh gel and preserved gel.
\end{abstract}

Keywords: Gel; Fastness; Dyes; Paste; Scattering; Absorption; Fabric

\section{Introduction}

Aloe plant belongs to Aloaceae family, and has more than 400 species. The species are easily recognized by their flower and rosettes, thick and succulent leaves, which are sometimes spotted. Most have leaves more or less $\mathrm{V}$-shaped in cross section. The leaf margin is almost always armed with sharp teeth. The different aloe species have different flowering periods; some flower over an extended period, thereby offering a continuous food supply for nectar feeding birds and insects. Each flower is supported by a bract, the shape and size of which are important for the identification of the species. Flower colouration is most often red, orange or yellow, and rarely white $[1,2]$.

Aloe species have been used for a long time in folk medicine for the treatment of constipation, burns and dermatitis. Recently, some species of Aloe have been used in a wide range of skin and hair care products, and also form the basis of health drinks and laxative drugs. The slimy gel inside the leaves consists of a complex mixture of polysaccharides, amino acids, minerals, trace elements and other biologically active substances, such as enzymes. Recent research has indicated that Aloe might kill the bacteria responsible for tuberculosis, Mycobacterium tuberculosis, and virus causing herpes genitals and inhibits growth of many common organisms such as yeasts, fungi, and bacteria infecting wounds [3].

Aloe debrana is known in Ethiopia and found in South Wollo, Dessie zuria and Kalu woreda, among other species in the genus Aloe. This plant need very less water for living and has resistance to diseases and insects. The Aloe debrana plant has long (up to 20 inches long and 5 inches wide) leaves. Each plant usually has 12-16 leaves and can be harvested every 6 to 8 weeks by removing 3 to 4 leaves per plant. The fresh parenchyma gel from the centre of the leaf is clear and shining [4].

Most areas in South Wollo, the land is a steep mountain slope and not well structured to plough for farmers and also highly exposed to erosion. Aloe debrana plant can grow in the place with eco-friendly and keep control of land conservation as well. The gel from this plant can be used for several purposes like dyeing fabrics.

Disperse dyes are mainly used in the dyeing and printing of polyester and its blended fabrics. Disperse print has excellent printing properties like fastness and colour strength and involves localized coloration. This is usually achieved by applying thickened pastes containing dyes or pigments onto a fabric surface according to a given colour design. In particular, the viscosity of a print paste is critical and determines the volume of paste transferred to the fabric and the degree to which it spreads on the surface fabric [2,5-7].

Textile printing is carried out from a paste preparation, which consists of the colouring materials (pigments/dyes), thickeners, binders (in pigment printing), and other ingredients. The thickeners control rheology or flow behaviour of the print formulation, which in turn controls presentation and migration resistance of the printed color thereby ensuring clear cut design and the right outline definition of prints $[8,9]$.

The present work was undertaken with the aim of determining the ideal disperse printing conditions for attaining high performance polyester and cotton fabric prints using Aloe debrana gel as a new thickening agent. Moreover, as well known Ethiopia is currently one of fast growing African country in Industry, especially in Textile. All industries import these thickening agents from abroad. So, we believe that using this plant material as a thickening agent has benefits for the country by decrease foreign currency, because of high dominancy of Aloe debrana plants in all high lands of Ethiopia this work the community can secure food by selling the plant to the nearby industries, and it is non toxic to the workers in the factories and environmental friendly.

*Corresponding author: Sisay Awoke, Department of Chemistry, College of Natural Science, Wollo University, Ethiopia, Tel. +251911956384; E-mail: sisgenetre@gmail.com

Received November 07, 2013; Accepted December 03, 2013; Published December 10, 2013

Citation: Awoke S, Adugna Y, Jihad R, Getaneh H (2013) The Importance of Aloe debrana Plant as a Thickening Agent for Disperse Printing of Polyester and Cotton in Textile Industry. J Textile Sci Eng 4: 147. doi:10.4172/2165-8064.1000147

Copyright: $\odot 2013$ Awoke S, et al. This is an open-access article distributed under the terms of the Creative Commons Attribution License, which permits unrestricted use, distribution, and reproduction in any medium, provided the original author and source are credited. 


\section{Materials and Methods}

\section{Description of study area}

The study areas were encompassing South Wollo Zone: Dessie zuria and Kalu woreda, and then two kebele from each woreda, Harawobelo and Harbu respectively were selected. The selection of kebele was depending on the dominant availability of Aloe debrana plants in that particular area (after conducting survey).

\section{Data collection}

The data collection was conducted in two phases and sampling method was purposive. In the first phase the survey was undertaken to identify the Aloe plant species based on their size and amount of the gel found inside. In the second phase, the plant species was collected from the selected areas forests of study areas. The amount of the gel found inside the Aloe debrana from Kalu was not enough for experimental use due to the hot season, as known the gel content is dependent on geographical location and weather condition. The sample collected from Dessie zuriya was good enough for experimental use due to high content of gel.

\section{Materials}

Bleached $100 \%$ polyester $\left(250 \mathrm{~g} / \mathrm{m}^{2}\right)$, and $100 \%$ cotton $\left(250 \mathrm{~g} / \mathrm{m}^{2}\right)$ woven fabrics were used.

Aloe debrana gel, Alginate and emulsion were used as thickening agent. The disperse dyes was Disperse Blue, and also chemicals; sodium bicarbonate, citric acid and urea were used.

\section{Methods}

Extraction of aloe gel: The gel of Aloe debrana was carefully extracted with the help of knife from the part of the plant as an ecofriendly and it was collected in the beaker. The beaker was immediately closed in order to protect it from contact of air, which is the cause of oxidation since the gel is sensitive. Using citric acid (lemon juice) as a natural preservative, the aloe extract was preserved for two months to evaluate the storing time.

Print paste and screen preparation: The printing pastes for disperse printing of polyester and Cotton was prepared by using fresh and preserved Aloe debran gel as a thickening agent. Each paste was prepared by using the following recipe: Disperse dye $30 \mathrm{~g} / \mathrm{Kg}$, Thickening agent $500 \mathrm{~g} / \mathrm{Kg}$, Urea $75 \mathrm{~g} / \mathrm{Kg}$, Citric acid $10 \mathrm{~g} / / \mathrm{Kg}$, Water $385 \mathrm{~mL} / \mathrm{Kg}$ as total weight of the paste was $1000 \mathrm{~g}$. Here when Aloe debrana fresh gel was being used as thickening agent the water from the recipe was omitted since there is more water in the gel naturally, thus the addition of extra water had done the paste thinner as compared with other and brought less effective the paste while printing. Totally the numbers of pastes prepared were seven and by using these pastes the printing was performed on cotton and polyester fabrics. In addition to the above pastes recipe, pure Aloe debrana without acid and without urea pastes were prepared with the same concentration of other chemicals and also another paste was prepared with mixture of the synthetic and Aloe debrana gel thickener but without changing the concentration and composition of other recipe. The flat screen was already prepared by Bahir Dar University Institute of Textile Engineering with Ethiopian beautiful woman picture.

Printing procedure and testing: Printing was carried out using the flat screen technique by squishing a total of $20 \mathrm{~g}$ paste four times manually and then printed samples were dried at $100^{\circ} \mathrm{C}$ for $3 \mathrm{~min}$ and fixed by mini-dryer and supper heated steam at $150^{\circ} \mathrm{C}$ for $6 \mathrm{~min}$. The samples were rinsed in cold water for $15 \mathrm{~min}$ and then in hot water at $60^{\circ} \mathrm{C}$ for $15 \mathrm{~min}$, followed by soaping with an anionic detergent $(2$ $\mathrm{g} / \mathrm{L}$ ), then rinsed well and finally dried at $85^{\circ} \mathrm{C}$ for $5 \mathrm{~min}$. The printed samples were evaluated for the depth of the prints (expressed as K/S, where $\mathrm{K}$ is the absorption coefficient and $\mathrm{S}$ is the scattering coefficient), as well as the fastness properties, e.g. washing and rubbing using standard methods.

Rubbing fastness and wash fastness: Color fastness to rubbing (dry and wet) was assessed manually by hand rubbing one sample ten times and grey scale as per ISO: 105-AO3-1995 extent of staining was used. Color fastness to washing of the dyed fabric samples was determined as per ISO: 105-AO2-1995 method using a launder-O-meter.

The $\mathrm{k} / \mathrm{s}$ value of printed sample was determined by measuring surface reflectance of the samples using a computer-aided Macbeth 2020 plus reflectance spectrophotometer, using the following Kubelka Munk equation with the help of relevant software:

$$
K / S=\frac{\left(1-R \lambda_{\max }\right)^{2}}{2 R \lambda_{\max }}
$$

where $\mathrm{K}$ the coefficient of absorption; $\mathrm{S}$ the coefficient of scattering and $\mathrm{R} \lambda \max$ the reflectance value of the sample at a particular wavelength, on which maximum absorption occurs for a particular dye/colour component.

\section{Results and Discussion}

From the selected areas based on different physical appearance (colour, leaf arrangement, height, edge, spot and stem) seven samples were taken to Addis Ababa herbarium. These samples were categorized into only two species, namely Aloe debrana and Aloe camperi. The two species have been planted in the garden of the herbarium and have been waiting for the flowering season of the plant to confirm their exact type with the help of flower. Since the ultimate goal of this study was to evaluate the performance of a thickening agent for disperse printing of polyester and cotton fabrics, as well as to search for the ideal printing paste components and fixation conditions for attaining darker prints with better overall fastness properties. Aloe debrana plant has been selected to precede this work since it has more gel compared from Aloe camperi.

From Table 1 it can be seen that using fresh Aloe debrana gel as a thickener of pastes to both cotton and polyester have good wash fastness, and rubbing fastness, the sample also have good colour staining fastness test because when they are compared with grey scale they show good result which means five. The decrement in $\mathrm{k} / \mathrm{s}$ is also small in both samples which mean there is minimum release of dyes from printed samples, this indicates the dye is reacted with the textile substrate and it is the desired property. The change of $\mathrm{k} / \mathrm{s}$ after washing indicates that there is an excess dyes on textile substrate before washing and the surface of printed sample have dull shade after washing which means there is an increase in brightness of printed sample so it indicates the decrement of $\mathrm{k} / \mathrm{s}$.

Alginate and Emulsion thickeners used as standard to compare the thickening potential of Aloe debrana. The change of $\mathrm{k} / \mathrm{s}$ after washing shows increment using Alginate and some decrement with Emulsion of both polyester and cotton fabrics. The nature of Alginate has harsh feeling so the surface of printed sample shows dull shade than that of Emulsion before washing. The wash fastness and rubbing result on printed polyester shows very good results whereas on cotton fabric the 
Citation: Awoke S, Adugna Y, Jihad R, Getaneh H (2013) The Importance of Aloe debrana Plant as a Thickening Agent for Disperse Printing of Polyester and Cotton in Textile Industry. J Textile Sci Eng 4: 147. doi:10.4172/2165-8064.1000147

Page 3 of 4

\begin{tabular}{|c|c|c|c|c|c|c|c|c|c|}
\hline \multirow[t]{2}{*}{ Print paste Thickener } & \multirow[t]{2}{*}{ Viscosity } & \multirow[t]{2}{*}{ Substrate } & \multicolumn{2}{|c|}{ K/S Value (400 nm) } & \multicolumn{2}{|c|}{ Wash Fastness } & \multicolumn{2}{|c|}{ Rubbing } & \multirow{2}{*}{ Handling } \\
\hline & & & Before Soaping & After Soaping & $\begin{array}{l}\text { Colour } \\
\text { Change }\end{array}$ & $\begin{array}{l}\text { Colour } \\
\text { Staining }\end{array}$ & Dry Rubbing & Wet Rubbing & \\
\hline \multirow[t]{2}{*}{ Aloe debrana } & \multirow[t]{2}{*}{10.6} & Polyester & 1.2299 & 1.1911 & 5 & 5 & 5 & 5 & $S$ \\
\hline & & Cotton & 0.4855 & 0.4028 & 5 & 5 & 5 & 5 & $S$ \\
\hline \multirow[t]{2}{*}{ Alginate } & \multirow[t]{2}{*}{11} & Polyester & 1.0607 & 1.0962 & 5 & 5 & 5 & 5 & $S$ \\
\hline & & Cotton & 0.1427 & 0.3411 & 2 & 2 & 2 & 2 & - \\
\hline \multirow[t]{2}{*}{ Emulsion } & \multirow[t]{2}{*}{11.4} & Polyester & 1.3161 & 1.1555 & 5 & 5 & 5 & 5 & $\mathrm{~S}$ \\
\hline & & Cotton & 0.7116 & 0.3153 & 2 & 2 & 2 & 2 & - \\
\hline \multirow[t]{2}{*}{ Aloe debrana-Alginate } & \multirow[t]{2}{*}{10.8} & Polyester & 1.0962 & 1.0705 & 5 & 5 & 5 & 5 & $S$ \\
\hline & & Cotton & 0.1715 & 0.2048 & 3 & 3 & 3 & 3 & - \\
\hline \multirow[t]{2}{*}{ Aloe debrana-Emulsion } & \multirow[t]{2}{*}{10.9} & Polyester & 2.6896 & 1.4238 & 5 & 5 & 5 & 5 & $S$ \\
\hline & & Cotton & 1.8151 & 0.4479 & 3 & 3 & 3 & 3 & - \\
\hline
\end{tabular}

Table 1: Pastes prepared with fresh Aloe debrana gel and other synthetic thickeners (Alginate and Emulsion).

\begin{tabular}{|c|c|c|c|c|c|c|c|c|c|}
\hline \multirow[t]{2}{*}{ Print paste Thickener } & \multirow[t]{2}{*}{ Viscosity } & \multirow[t]{2}{*}{ Substrate } & \multicolumn{2}{|c|}{$\mathrm{K} / \mathrm{S}$ Value $(400 \mathrm{~nm})$} & \multicolumn{2}{|c|}{ Wash Fastness } & \multicolumn{2}{|c|}{ Rubbing } & \multirow{2}{*}{ Handling } \\
\hline & & & Before Soaping & After Soaping & $\begin{array}{l}\text { Colour } \\
\text { Change }\end{array}$ & $\begin{array}{l}\text { Colour } \\
\text { Staining }\end{array}$ & Dry Rubbing & Wet Rubbing & \\
\hline \multirow[t]{2}{*}{ Aloe debrana } & \multirow[t]{2}{*}{10.7} & Polyester & 3.8369 & 1.2033 & 5 & 5 & 5 & 5 & S \\
\hline & & Cotton & 3.0815 & 1.5336 & 5 & 5 & 5 & 5 & S \\
\hline
\end{tabular}

Table 2: Printing paste prepared with preserved Aloe debrana gel.

\begin{tabular}{|c|c|c|c|c|c|c|c|c|c|}
\hline \multirow[t]{2}{*}{ Print paste Thickener } & \multirow[t]{2}{*}{ Viscosity } & \multirow[t]{2}{*}{ Substrate } & \multicolumn{2}{|c|}{$\mathrm{K} / \mathrm{S}$ Value $(400 \mathrm{~nm})$} & \multicolumn{2}{|c|}{ Wash Fastness } & \multicolumn{2}{|c|}{ Rubbing } & \multirow{2}{*}{ Handling } \\
\hline & & & Before Soaping & After Soaping & $\begin{array}{l}\text { Colour } \\
\text { Change }\end{array}$ & $\begin{array}{l}\text { Colour } \\
\text { Staining }\end{array}$ & Dry Rubbing & Wet Rubbing & \\
\hline \multirow{2}{*}{$\begin{array}{l}\text { Aloe debrana } \\
\text { without acid }\end{array}$} & \multirow[t]{2}{*}{10.6} & Polyester & 2.3033 & 1.6121 & $4 / 5$ & $4 / 5$ & $4 / 5$ & $4 / 5$ & $\mathrm{~m}$ \\
\hline & & Cotton & 2.9316 & 1.1532 & 4 & 4 & 4 & 4 & $\mathrm{~m}$ \\
\hline \multirow{2}{*}{$\begin{array}{l}\text { Aloe debrana } \\
\text { without Urea }\end{array}$} & \multirow[t]{2}{*}{10.6} & Polyester & 5.9613 & 4.9388 & $4 / 5$ & $4 / 5$ & $4 / 5$ & $4 / 5$ & $\mathrm{~m}$ \\
\hline & & Cotton & 2.4149 & 1.0306 & $4 / 5$ & $4 / 5$ & $4 / 5$ & $4 / 5$ & $\mathrm{~m}$ \\
\hline
\end{tabular}

Table 3: Printing paste prepared without acid and without urea using fresh Aloe debrana gel.

result is very poor. That is may be the fabric nature of polyester has hydrophobic end chemically and cotton has hydrophilic chemical end and the dye is hydrophobic which brought good chemical interaction with polyester and less with cotton. However the mixture of Aloe debrana with Alginate and Emulsion (Aloe debrana-Emulsion and Aloe debrana-Alginate) showed some change of $\mathrm{k} / \mathrm{s}$ value after washing and there is some improvement of the wash fastness and rubbing on cotton when it is compared to that of pure alginate and emulsion, this indicates that Aloe debrana gel has a potential of changing the property of disperse dye to have good interaction with cotton.

The viscosity of Aloe debrana paste is good enough when compared with synthetic thickeners. Moreover, the viscosity of natural thickeners, Aloe debrana, improved when small amount of synthetic thickeners are added.

The gel was stored for two months within highly secured container by using lemon juice as preservative. The result in Table 2 indicated that the decrement in value of $\mathrm{k} / \mathrm{s}$ of preserved Aloe debrana gel used for printing of cotton and polyester fabric is because of a removal of some un reacted dyes from the surface of the fabric which results to the increment of reflectance of the fabric. The sample has good fastness to washing and rubbing, it also shows good result in colour staining test. The comparison of the $\mathrm{k} / \mathrm{s}$ value of preserved gel with fresh Aloe debrana increased that indicates the viscosity of the preserved gel is better than fresh gel. Even though the change in $\mathrm{k} / \mathrm{s}$ value in Table 3 is the same trend with normal recipe of Aloe debrana, the result of washing and rubbing fastness was poor. This shows the presence of the acid and urea in the recipe improves the media and hygroscopic nature of the paste, respectively, which are very essential component of the print paste. The absences of both acid and urea do not affect the viscosity of the paste.

\section{Heating temperature, fixation time and stirring time}

Increasing heating temperature from $140^{\circ} \mathrm{C}$ up to $180^{\circ} \mathrm{C}$ was accompanied by a gradual sharp increase in the depth of the obtained disperse prints, facilitating the release of disperse dye from the thickener film, allowing sublimation and diffusion into the accessible area of the polyester and cotton structure, as well as enhancing the extent of dye retention and fixation, thereby giving rise to a more intense depth of shade and increase the swell ability of both the thickener film and the polyester structure.

The effect of heating fixation time on the extent of dyeing polyester and cotton fabrics is clear that prolonging the heating time up to $6 \mathrm{~min}$ at $180^{\circ} \mathrm{C}$ resulted in a remarkable increase in the depth of the obtained disperse prints. Decreasing in $\mathrm{k} / \mathrm{s}$ value indicates that improvement of scattering of light after wash. That reflected the positive role of proper heating time on swelling the thickening film, enhancing the extent of release of dye molecules from the thickener film as well as dye sublimation, adsorption onto and diffusion within the substrate, thereby enabling the volatile components and dye vapour to be strongly adsorbed and retained by the hydrophobic polyester and hydrophilic cotton component. Expanded fixation time may results migration on cotton fabrics and fire hazard for polyester fabrics.

Stirring is the process of mixing the components of print paste; thickener, urea, acetic acid, disperse blue and water, to make solid paste. When the time of stirring increase the paste becomes uniform and good for printing, but when the stirring time is extended the paste prepared from Aloe debrana becomes enough solid for good performance or viscosity for printing whereas the synthetic thickeners, alginate and emulsion, becomes more solid which is not good for printing. 
Citation: Awoke S, Adugna Y, Jihad R, Getaneh H (2013) The Importance of Aloe debrana Plant as a Thickening Agent for Disperse Printing of Polyester and Cotton in Textile Industry. J Textile Sci Eng 4: 147. doi:10.4172/2165-8064.1000147

\section{Effect of substrate and thickeners}

Changes in the $\mathrm{k} / \mathrm{s}$ values of the obtained prints along with their fastness properties as a function of type of substrate (fabric) are shown on tables, which demonstrates that the depth of the obtained prints was governed by the kind of substrate, its chemical structure, and its affinity for the used dye, the degree of penetration, interaction and subsequent fixation. Polyester and cotton have different nature chemically, the former is the polymer of non polar (hydrophobic) and the latter is polymer of polar (hydrophilic). Aloe debrana as a thickening agent in disperse printing results in good thickening efficiency as well as better depth and fastness properties of the obtained prints in comparison with the Alginate and Emulsion thickener.

\section{Effect of gel concentration}

Increasing the thickening agent (Aloe debrana) concentration from $300 \mathrm{~g} / \mathrm{kg}$ up to $500 \mathrm{~g} / \mathrm{kg}$ resulted in an improvement in the $\mathrm{k} / \mathrm{s}$ values of the disperse prints, which could be discussed in terms of higher paste viscosity. Further increase in the concentration of Aloe debrana gel had no negative effect on the depth of the obtained prints and softness. The overall fastness properties of the printed polyester fabric samples showed that using Aloe debrana at $300 \mathrm{~g} / \mathrm{Kg}$ as a thickening agent yielded the best improvement in the handling, sharpness and fastness properties of the printed samples. However, in the case of cotton fabrics numerically show less $\mathrm{k} / \mathrm{s}$ than that of polyester it might be the chemical affinity of the gel with the cotton product, but still the gray scale and handling show the same result.

\section{Conclusion}

This research focused on the application of a new thickening agent based on Aloe debrana gel for disperse printing of polyester and cotton. On the basis of the experimental results, we have made the following conclusions: Changes in the $\mathrm{k} / \mathrm{s}$ values of the obtained prints along with their fastness properties as a function of type of substrate (fabric) are shown, which demonstrates that the depth of the obtained prints was governed by the kind of substrate, its chemical structure, and its affinity for the used dye, the degree of penetration, interaction and subsequent fixation. Polyester and cotton have different nature chemically. Cotton has hydrophilic end chemical nature and it is not expected to have good interaction with hydrophobic dyes however using the Aloe debrana thickener with the hydrophobic dyes like disperse dyes showed very good interaction with cotton and printing was carried out with good performance. A natural preservative lemon juice has high potential to preserve the gel more than two months. The results showed that no significant difference between fresh gel and preserved gel. Apart from this, the chemical aspects of the gel with dye and cotton fabric need further investigation.

\section{Acknowledgement}

First of all, we would like to thank Wollo University, which provides conducive environment and budget to do this work without any problem. We are also pleased to thank South Wollo Zone Agriculture office, Bahir Dar University of Textile Institute, AddisAbaba University Herberium center. Lastly we also acknowledge al respected Departments for their cooperativeness during the work.

\section{References}

1. Nordal I, Sebsebe D, Stabbetorp O (1998) Endemism in groups of Ethiopian geophytes ('Liliiflorae'). Food and Agricultural Organization 247-258.

2. Ragheb AA (1994) Suitability of Nitfogen-Containing starch derivative in printing polyester fabrics with disperse dyes. American Dyestuff Reporter 83: 15-24.

3. Vazquez B, Avila G, Segura D, Escalante B (1996) Antiinflammatory activity of extracts from Aloe vera gel. J Ethnopharmacol 55: 69-75.

4. Dagne E, Bisrat D, Viljoen A, Van Wyk B (2000) Chemistry of Aloe species Curr Org Chem 4: 1055-1078.

5. Enas MR, El Zairy (2011) New thickening agent based on aloe vera gel for disperse printing of polyester. AUTEX Res J 11

6. Aspland JR (1997) Textile dyeing and coloration. AATCC, NC, US.

7. Ibrahim NA, El Zairy EMR (2009) Union disperse printing and UV-protecting of Wool/polyester blend using a reactive ß-cyclodextrin. Carb Polymers 76: 244 249 .

8. Choudhury AKR (2006) Textile preparation and dyeing. Science publishers, Enfield, $\mathrm{NH}$, USA.

9. Ibrahim NA, El Zairy MR, Abo-Shosha MH (1994) Preparation and rheology of new synthetic thickeners based on polyacrylic acid. Dyes and Pigments 24 249-257. 